\title{
EL APORTE A LA SALUD PÚBLICA DEL DR. ABRAHAM HORWITZ: LUCES DESDE LA CONTEMPORANEIDAD
}

Jorge Jiménez de la Jara ${ }^{1}$, Lorenzo Agar Corbinos $^{2}$, Nicole Saffie Guevara ${ }^{3}$

Resumen: El propósito de este artículo es revisar las ideas centrales del pensamiento del Dr. Abraham Horwitz (1910-2000), quien se dedicó en cuerpo y alma a la salud pública, tanto de Chile como de América Latina y el Caribe. Este trabajo aborda su pensamiento desde una perspectiva contemporánea. Este es justamente nuestro desafío. Aunque vivimos en un mundo diferente al de los años sesenta, con grandes progresos en materia de salud, aún existen enormes brechas entre países y grupos sociales, además de grandes transformaciones sociodemográficas y económicas con alto impacto social.

Palabras clave: salud pública, medicina social, epidemiología, equidad, ética, educación, desarrollo

The contribution to public health of Abraham Horwitz: insigths from contemporaneous times

Abstract: The purpose of this article is to review the main ideas of doctor Abraham Horwitz's thinking (1910-2000), who dedicated all his life to public health, both in Chile and Latin America and Caribbean. This paper focuses his thought from a contemporary perspective. This is precisely our challenge. Although we live in a different world from the sixties, with great progress in health, there are still huge gaps between countries and social groups, as well as large demographic and economic transformations with high social impact.

Key words: public health, social medicine, epidemiology, equity, ethics, education, development

\section{A contribuição à saúde pública do Dr. Abraham Horwitz: luzes a partir da contemporaneidade}

Resumo: O propósito deste artigo é revisar as ideias centrais do pensamento do Dr. Abraham Horwitz (1910-2000), quem se dedicou corpo e alma à saúde pública, tanto do Chile como da América Latina e do Caribe. Este trabalho aborda o seu pensamento a partir de uma perspectiva contemporânea. Este é justamente o nosso desafio. Embora vivamos num mundo diferente ao dos anos sessenta, com grandes progressos em matéria de saúde, ainda existem enormes diferenças entre países e grupos sociais, além de grandes transformaçôes sociodemográficas e econômicas com alto impacto social.

Palavras-chave: saúde pública, medicina social, epidemiologia, equidade, ética, educação, desenvolvimento

\footnotetext{
${ }^{1}$ Profesor titular de la Pontificia Universidad Católica de Chile, Chile

${ }^{2}$ Profesor Agregado de la Escuela de Salud Pública, Facultad de Medicina, Universidad de Chile, Chile Correspondencia: lorenzoagar@gmail.com

${ }^{3}$ Periodista de la Vicerrectoría de Comunicaciones y Educación Continua, Pontificia Universidad Católica de Chile, Chile
} 
Quisiera ver que "salud para todos" se hiciera realidad, cualquiera sea el mecanismo, cualquiera el modo de acción. Me doy cuenta que no es sencillo. Procurar salud para todos es tarea de todo tiempo, no necesariamente para este nuevo milenio. No es una empresa exclusiva para los médicos. La salud es una responsabilidad social, integral y en la que todos debemos participar en forma armoniosa. (Dr. Abraham Horwitz Barak).

\section{Introducción}

Abraham Horwitz (1910-2000), infectólogo y salubrista, creó la Escuela de Salubridad de la Universidad de Chile e hizo un valioso aporte normativo al Servicio Nacional de Salud. A los 47 años fue nombrado director de la Organización Panamericana de la Salud (OPS), manteniéndose en el cargo por 16 ańos, entre 1958 y 1975 . Este fue un verdadero hito, no solo por ser el primer latinoamericano en el puesto, sino que a partir de su mandato cambió la concepción misma de la salud y la cooperación técnica internacional. Durante su gestión impulsó un gran desarrollo institucional, especialmente en los temas de saneamiento básico, nutrición y erradicación de enfermedades transmisibles, abriendo líneas de pensamiento inexploradas, tales como las relaciones entre economía, administración moderna y salud. Asimismo, esta época estuvo marcada por profundos cambios sociales, políticos y económicos, los que generaron un ambiente propicio para la búsqueda de nuevas respuestas y soluciones para los problemas que aquejaban a los países de la región.

Este trabajo aborda su pensamiento desde una perspectiva contemporánea. Este es justamente nuestro desafío. Aunque vivimos en un mundo diferente al de los años sesenta, con grandes progresos en materia de salud, aún existen enormes brechas entre países y grupos sociales, además de grandes transformaciones sociodemográficas y económicas con alto impacto social.

Ya en el nuevo milenio, las ideas del Dr. Horwitz siguen teniendo plena vigencia, especialmente su enfoque sobre la necesidad de una visión comprensiva de diversas disciplinas en torno a la salud. Es imperioso, enfatizaba, que se produzca interacción y búsqueda de aquellas convergencias que reconozcan el vínculo entre la dolencia individual y los aspectos relevantes del entorno social. Esta mirada, derivada de la modernidad y de la primacía de la racionalidad y el pensamiento empírico dominante en Occidente durante los años de su vida laboral, encuentra en el Dr. Horwitz un claro matiz.

\section{Nace el joven médico}

Eran los años de las grandes migraciones europeas hacia las Américas, siendo Estados Unidos y Argentina los destinos predilectos. Las crisis políticas, las hambrunas y las guerras motivaron, especialmente a los jóvenes, a aventurarse hacia territorios inexplorados y florecientes, llenos de oportunidades. Incluso familias y pueblos enteros se sumaron a estas expediciones, huyendo de los "pogromos" zaristas o grandes persecuciones al pueblo judío en la Rusia imperial, en busca de la paz.

Entre ellos estaban los Horwitz-Barak, originarios de la aldea de Jotin en la actual República de Moldavia, quienes deciden dejar su tierra en búsqueda de un futuro mejor. Llegan a Chile luego de muchas peripecias, como tantos otros inmigrantes. Abraham nace en Santiago de Chile el 25 de diciembre de 1910, año del primer centenario de la independencia. Quizás una coincidencia que presagiaba que con él nacería una nueva forma de vislumbrar la salud pública del continente(1).

El brillante joven recibe su título de médico en 1936. Era la época en que la tuberculosis, la sífilis, el tifus exantemático, el sarampión y decenas de otras patologías infectocontagiosas, eran la causa de dos tercios de las muertes de nińos y adultos jóvenes. Decidirse por esta área de la medicina reflejaba una correcta visión de conjunto y, sobre todo, un alto grado de coraje. Más tarde, refiriéndose a su cargo como director de la OPS, Horwitz se enorgullecía por la enorme oportunidad que ofrecía el cargo para servir desde la salud pública a todos los países de las Américas, mejorando sus condiciones de vida.

En 1942 obtiene una beca de la Fundación Rockefeller para desempeñarse, hasta septiembre de 1943, como médico residente en el Hospital de 
Enfermedades Infecciosas Herman Kiefer de Detroit (Michigan, Estados Unidos), especializándose en enfermedades infecciosas. Luego, realiza su Master of Public Health (Magíster en Salud Pública) en la prestigiosa Universidad de Johns Hopkins de Baltimore (Maryland, Estados Unidos), grado que recibe en 1944(1).

\section{La Escuela de Salubridad de la Universidad de Chile}

A su regreso a Chile, Abraham Horwitz tiene en mente el objetivo de organizar la Escuela de Salubridad en la Universidad de Chile, con el apoyo de la Fundación Rockefeller. Después del profesor Hernán Romero, se convierte de hecho en su segundo director entre 1945 y 1947 (1). Su visionaria mirada sobre lo que debía ser la medicina en Chile y Latinoamérica se hizo realidad a través de esta institución que, con el paso de los años, se constituyó en uno de los pilares de la medicina social chilena, por su capacidad para investigar y formar recursos humanos de alto nivel.

En forma constante, el Dr. Horwitz subraya que la salud no pertenece exclusivamente a los médicos, sino que es un producto social de responsabilidad compartida. Y hace ver que es allí en donde reside la dificultad, puesto que se necesita la cooperación de distintas instancias no ligadas estrictamente a la salud, como por ejemplo las condiciones del medio ambiente, la educación, el trabajo y la vivienda. Insiste en que los principios en que se basa la epidemiología han permanecido inalterables; lo que ha variado son las circunstancias del entorno social y ambiental que posibilitan o no su ocurrencia(2).

El prestigio alcanzado por el Dr. Horwitz no solamente brilló en Chile, sino que traspasó las fronteras. En 1950 fue llamado a trabajar para la Organización Panamericana de la Salud.

\section{Nace el Servicio Nacional de Salud (SNS)}

En 1939, un terremoto sacudió Chillán, devastando el centro sur del país. Entonces, un grupo de sanitaristas y políticos intuitivos comenzó a discutir la unificación de los servicios de atención de salud en una sola estructura. Sin embargo, esta reforma recién vio la luz en 1952, con la aproba- ción de la Ley 10.383 que daba origen al Servicio Nacional de Salud (SNS), no sin cierta resistencia en algunos sectores. Fue el gran año de la salud pública chilena y, por supuesto, allí estaba el Dr. Abraham Horwitz, dispuesto a cumplir la tarea de organizar esta nueva institución, labor que cumplió durante seis fructíferos años.

Estructuralmente, el SNS integra verticalmente las funciones de financiamiento y provisión de servicios de salud. También genera la colaboración entre la asistencia y la docencia e investigación, en estrecha relación con la Facultad de Medicina de la Universidad de Chile. La integración de 15 plantas de personal distintas tomó tres años, mientras que la creación de instrumentos y doctrinas claras para la acción fue larga y compleja. "La estrategia de Abraham Horwitz fue centralización en la norma y descentralización en la acción; es decir, tener normas claras pero que los médicos contaran con suficiente libertad y discrecionalidad para aplicarlas", explica en entrevista su sobrino, el también médico Isidoro Horwitz ${ }^{4}$.

La estrategia para llegar hasta los sectores rurales y de provincias se materializó gracias a una solución conjunta entre el SNS y la Facultad de Medicina: el ciclo de destinación, es decir, la creación de los médicos generales de zona y residencia de especialidades.

Hacia 1957, el SNS ya tenía los cimientos necesarios para convertirse en una institución sólida y eficaz de la medicina social chilena, y sus creadores empezaron a mirar hacia otros destinos. A los 47 años Abraham Horwitz, tenía el suyo ya trazado.

Cuando el Dr. Horwitz fue nombrado en 1958 director general de la Oficina Sanitaria Panamericana (OPS), no solo asumió un gran desafío, sino que se transformó en el primer chileno, y latinoamericano, en ocupar este cargo. Fue un verdadero hito, ya que, siguiendo la lógica de Guerra Fría de la época, en que el mundo estaba dividido en dos bloques liderados por Estados Unidos y la Unión Soviética respectivamente, resultaba al menos curioso que la nueva cabeza de este organismo, que agrupaba a los países americanos, no fuera un

${ }^{4}$ Saffie N. Entrevista al Dr. Isidoro Horwitz. Santiago de Chile, octubre, 2010. 
estadounidense, al igual que sus antecesores. De esta manera, se convertía en una especie de asesor de los ministros de salud que regían los destinos de unas 400 millones de personas.

Pero el panorama no era fácil. El mundo estaba convulsionado: los estudiantes protestaban por las injusticias sociales, los medioambientalistas alertaban por el uso inadecuado de productos químicos en los campos, los guerrilleros libraban múltiples campos de batalla y la expansión económica se detenía, dando paso a una inflación mundial que disparó los precios de los alimentos, combustibles y materiales, ampliando las diferencias entre países ricos y pobres. A comienzos de los sesenta, los gobiernos de las Américas empezaron a darse cuenta que debían aprovechar las nuevas tecnologías para incrementar los niveles de vida de la población. En 1961, los países miembros de la Organización de Estados Americanos (OEA) definieron los objetivos para sus naciones: abastecimiento de agua y saneamiento, reducción de la mortalidad infantil, control de las enfermedades transmisibles, mejoras en la nutrición, capacitación y desarrollo personal de salud, fortalecimiento de los servicios básicos e intensificación de la investigación científica, utilizando el conocimiento para evitar y curar las enfermedades. Los gobiernos reconocieron que la salud era un factor fundamental para el desarrollo económico y social.

Durante su mandato, el Dr. Horwitz hizo enormes contribuciones a la salud pública del continente. Los mayores esfuerzos estuvieron centrados en el combate de las enfermedades, tanto de aquellas prevenibles mediante vacunas, como de las que requerían otros métodos de prevención y control(3).

\section{Las claves del pensamiento de Abraham Horwitz}

En los años sesenta, el paradigma de la modernidad mostraba una enorme fuerza. $Y$ es por eso que el pensamiento de este médico visionario nos alimenta de una mirada innovadora y digna de ser rescatada, divulgada y actualizada. Al respecto, Jorge Larraín afirma: "Mientras la modernidad creía en el progreso lineal, la tecnología, la ciencia positiva y la razón, la posmodernidad privilegia la indeterminación, la fragmentación, la heterogeneidad y la diferencia. Desconfía de las verdades absolutas y de las metas narrativas o discursos totalizantes de aplicación universal, especialmente de aquellos que proponen la emancipación humana" (4:244).

Durante el mandato del Dr. Horwitz en la OPS, Latinoamérica vivía una época de crisis social y política. En la década de los sesenta los países plenamente democráticos eran pocos, existían muchas dictaduras o gobiernos militares autoritarios, a lo que se sumaba las guerrillas de liberación nacional. Panorama que no hacía nada fácil la ejecución de los planes y campañas nacionales de salud.

El Dr. Horwitz plantea con profunda agudeza la necesidad de incluir las sensibilidades y pluralidad de experiencias de los diversos actores al abordar los problemas sociales que afectaban la salud humana. Como insiste en todos sus textos y discursos, la salud es un producto social cuya responsabilidad debe ser compartida por todos. Por tal razón, se requiere el aporte de diversas esferas disciplinarias que interactúen tanto con aspectos sanitarios estructurales como con elementos de la salud propios de cada individuo. La complementariedad de las miradas, incluida por cierto la del mismo sujeto afectado, resulta fundamental para un buen diagnóstico y tratamiento efectivo.

La bioética enfatiza las miradas interdisciplinarias en las formas de intervenir y tomar decisiones en la salud de las personas. En los inicios de los años setenta el Dr. Potter, padre de esta disciplina, advierte acerca del profundo divorcio entre el paradigma científico, rodeado de un aura empírico, y las ciencias sociales. Nos plantea en aquella época la imperiosa necesidad de que ambas miradas se encuentren. Es precisamente la bioética la que hace un llamado a que las investigaciones científicas incorporen un componente ético, tanto en el impacto humano como el entorno ambiental.

La visión bioética, de amplio desarrollo en nuestros días, se encuentra con lo enunciado por el Dr. Horwitz en el sentido de la responsabilidad social; y nos atrevemos a pensar que también apunta a la responsabilidad individual. Es decir, el individuo y su contexto (la familia), el individuo en su con- 
texto (sistema de salud) y el individuo con su contexto (cercanos relevantes). A eso hace referencia, seguramente, cuando habla de que todos debemos participar en la salud en forma armoniosa.

El Dr. Horwitz nos presenta un abordaje polifacético, dinámico e interdependiente, que responde a riesgos locales y globales en salud, y que tiene como ejes la equidad, la ética y el respeto a los derechos humanos. Incluso los actuales planteamientos, que hablan de salud global ligada a la justicia social y como un derecho universal, recogen sin duda la semilla de sus ideas.

El reconocimiento creciente de las técnicas cualitativas de análisis social es una forma de aceptar que, junto con las perspectivas que han legado las ciencias duras a las ciencias humanas, las ciencias de la salud hoy en día también se han abierto al reconocimiento de los enfoques psicosociales. Y en este reconocimiento la obra del Dr. Horwitz ha hecho un aporte pionero.

El Dr. Horwitz analizó las interrelaciones de la salud y el desarrollo, con la economía y la educación. Estuvo un paso adelante al poner de manifiesto que el desarrollo no es solamente un asunto derivado de los ingresos o de la capacidad productiva e industrial de las naciones, sino que la educación juega un rol clave, acercándose al concepto de desarrollo humano de los años noventa.

Las ideas centrales de editoriales y otros documentos publicados por el Dr. Horwitz entre 1958 y 1970, muestran una permanente legitimación de América Latina y el Caribe como un bloque sólido al interior del continente. Sus planteamientos representan a la región globalmente, sin hacer comparaciones locales, colocando un interesante horizonte de unidad regional, lo que todavía es un desafío.

$\mathrm{Su}$ enfoque intenta aunar las distintas realidades nacionales en una unidad regional. Suele presentar, desarrollar y validar temas primordiales para la región. Una vez que estos temas han sido planteados y analizados independientemente, procede a contactarlos e integrarlos globalmente. Este trabajo permite observar la interrelación de problemas de distinta índole, con la conciencia de que los problemas de salud no son aislados ni aislables, sino que se presentan en un contexto determinado. Es de esa forma como deben ser abordados.

\section{En torno a la epidemiología}

Para el Dr. Horwitz, la epidemiología era una herramienta esencial para avanzar en salud pública, de ahí que hiciera grandes esfuerzos para promover su desarrollo en las Américas. Estaba convencido de que los principios de esta disciplina han permanecido inalterables, lo que cambia es el ambiente y la manera en que los seres vivos se adaptan a él, lo que determina las enfermedades. La epidemiología se ha convertido en una disciplina sumamente dinámica, la que ha hecho uso de muchos de los desarrollos tecnológicos y conceptuales de las últimas décadas.

Horwitz cree que no son pocos los problemas de salud en las Américas cuya solución debe buscarse por procedimientos epidemiológicos. Dada la variación geográfica de la enfermedad, hace notar que no hay campo en que esta disciplina no tenga un papel importante que desempeñar. En su artículo "La epidemiología en América Latina", recomienda incorporar materias epidemiológicas en la preparación y formación de los futuros médicos. De hecho, impulsa la investigación práctica en escuelas de medicina, para que se conviertan en verdaderos centros de adiestramiento en esta área(5:191-194). En los albores de la década de los sesenta, plantea que en muchos países de la región se produce una suerte de polarización epidemiológica, donde persisten las enfermedades transmisibles, y los problemas crónicos ocupan un espacio creciente y de importancia crítica. Esta situación continúa planteando grandes desafíos en la actualidad.

En aquella época se inicia un creciente proceso de urbanización en los países de América Latina y el Caribe. A diferencia de las naciones desarrolladas de Europa Occidental, en nuestra latitud la implosión urbana no fue acompañada de un proceso correlativo de industrialización. De ahí derivó un crecimiento urbano anacrónico, producto de migraciones internas campo-ciudad, que están en el origen de las grandes periferias de marginalidad que hasta nuestros días acompañan a muchas de las metrópolis de la región. El Dr. Horwitz estaba muy consciente de este proceso, pues hacía no- 
tar que todo análisis debía considerar que “(...) el fenómeno más significativo que acompaña el crecimiento económico ha sido el traslado de la población trabajadora de la agricultura a la industria, y de esta al comercio y los servicios" (6:100).

Con todo, la urbanización y el control de las enfermedades que atacaban esencialmente en las edades tempranas, entre otros factores, produjeron un rápido aumento en la esperanza de vida al nacer. Como bien sabemos, la natalidad, que depende mayormente de variables ligadas a la cultura, se mantuvo en niveles altos durante las primeras décadas de la segunda mitad del siglo XX. De ahí que el crecimiento de la población haya sido en este lapso el más alto registrado en la historia humana.

Se constatan pues grandes cambios sociales. El Dr. Horwitz pone énfasis en que existe un analfabetismo extendido, ingresos per cápita que oscilan entre los 100 y 500 dólares al año, la dependencia de la exportación de un solo producto e inversiones insuficientes. Las inevitables consecuencias de todo ello, dice, son la pobreza, la miseria y la marginalidad urbana.

Por cierto que los problemas de salud cambiaron. Así como también empezaron a modificarse las expectativas de la población en torno a su calidad de vida, lo cual generó una fuerte presión sobre la medicina y el sistema de salud. El Dr. Horwitz pone énfasis en que muchos problemas de salud en nuestra región tienen relación con la variación geográfica de la enfermedad. Afirma que para poder avanzar correctamente en el trabajo epidemiológico, se debe perfeccionar el registro y las estadísticas vitales. Así lo expresaba al prologar el clásico estudio de la mortalidad en la infancia, de Puffer y Serrano5 (1973), cuando afirmaba que las estadísticas oficiales no alcanzaban a revelar la real magnitud de los problemas de salud y que se hacía necesario intensificar los esfuerzos para la formación de estadísticos y auxiliares con el fin de mejorar la calidad de los datos, usar en forma más racional los recursos y evaluar las acciones y efectos sociales logrados. En su artículo "La epidemiología en la América Latina", hace ver que “(...) con el conocimiento de, por ejemplo, la

\footnotetext{
${ }^{5}$ Es importante destacar que un trabajo de estas características no ha sido repetido hasta nuestros días.
}

distribución de la mortalidad —a través de las tasas de defunción, por grupos de edad o correspondientes a enfermedades específicas - los investigadores empezarán a explorar los factores causantes de las diferencias que se descubran (...). Este tipo de investigación coordinada permitirá que ciertas escuelas de medicina seleccionadas se conviertan en centros de investigación práctica y faciliten un buen adiestramiento en epidemiología" (5:192).

Esto significó favorecer el desarrollo de la epidemiología, definida por el Dr. Horwitz como una ciencia que comprende todas las relaciones del hombre con el ambiente que lo rodea, tanto mediato como inmediato, esté sano o enfermo. Concepto plenamente vigente hoy en día. Asimismo, promovió la institución de esta disciplina científica como elemento esencial para el desarrollo de políticas sanitarias, especialmente en materia de salud preventiva; e insistía en que eran muchos los problemas de salud en las Américas cuya solución debía buscarse por medio de procedimientos epidemiológicos.

En 1961, durante el simposio sobre medicina mundial en la Universidad de Yale, el Dr. Horwitz presentó las tres áreas consideraba primordiales para la práctica epidemiológica, que llegaron a ser los ejes del desarrollo de la epidemiología en el continente durante las últimas décadas: servicios de salud, investigación y capacitación en epidemiología. A partir de estas tres perspectivas fue definida la estrategia de cooperación de la OPS para el fortalecimiento de la capacidad nacional en epidemiología de los países de la región(7).

El Dr. Horwitz fue un precursor de la cooperación entre países en materia de investigación, impulsando el estudio comparado y promoviendo el desarrollo de un "mercado común intelectual", como él mismo lo llamó. El resultado fue el surgimiento de estudios multicéntricos en temas de mortalidad infantil, enfermedades infecciosas y otras.

Más allá de la imperiosa necesidad de realizar investigaciones epidemiológicas respecto de la atención médica, el Dr. Horwitz considera urgente evaluar las necesidades en servicios de salud para la planificación de programas a largo plazo. Men- 
ciona entre algunas enfermedades "modernas", o de la época, las virales, afecciones mentales, el alcoholismo, las radiaciones y la polución atmosférica, plenamente vigentes.

En distintos escritos, entre 1962 y 1963, destaca la relevancia que adquiere la salud mental ante los cambios que conlleva la migración de las áreas rurales a las urbanas en América Latina. Horwitz advierte que las transformaciones de las condiciones de vida en los aspectos biológico, ideológico, político, económico y social, hacen surgir una serie de problemas sicológicos, al tratar de adaptarse a una forma de vida muy distinta. El crecimiento demográfico que no va acompañado del correspondiente incremento de recursos, da lugar a una desproporción cada vez mayor entre los deseos de una población de acceder a bienes y servicios - y que tiene un mayor sentido del progreso y la igualdad-y las posibilidades reales de satisfacer esa demanda. Subraya que los fenómenos de la industrialización y urbanización plantean conflictos en los individuos que modifican tanto los sistemas de valores éticos, como los papeles tradicionales de la autoridad del hombre y la mujer, transformando la dinámica familiar.

Estos planteamientos sorprendían en la época, ya que aún no se vislumbraba con claridad las interrelaciones entre demografía y desarrollo. Recién en la Conferencia de Población de 1984, en México, se comienza a reconocer la estrecha interdependencia, por ejemplo, entre el crecimiento demográfico, los niveles de natalidad y mortalidad, y la estructura por edades, con variables ligadas a la educación, economía, localización espacial y el rol de la mujer, entre otros. Sin duda que el pensamiento del Dr. Horwitz contribuyó en esta reflexión, siendo uno de sus principales aportes.

En el campo epidemiológico aparecen los vínculos estrechos entre las enfermedades físicas y mentales con las condiciones ambientales, especialmente respecto de las condiciones de vida en las grandes ciudades que presentan tasas elevadas de contaminación ambiental y acústica. A esto se suma el cambio climático, contribuyendo a expandir los factores causales de muchas de las enfermedades que hoy padecemos.
Pese a la importancia de estos temas, estos no recibían la suficiente atención durante la formación de los médicos ni del personal de salud pública, algo que hizo ver claramente el Dr. Horwitz.

Afirma: "El elemento predominante en la enseńanza médica sigue siendo, al parecer, el diagnóstico y el tratamiento de enfermedades. No se dedica debida atención a la medicina como ciencia social, ni al estudio del hombre como ente biológico y social (...). Por consiguiente, el estudiante no se forma un concepto de la medicina como una ciencia que armoniza la prevención de las enfermedades, el tratamiento de los enfermos y el fomento de la salud" (7:4).

Hace hincapié en la necesidad de realizar un estudio en el campo de la salud pública y la epidemiología, la cual se ha concentrado fundamentalmente en el control de las enfermedades transmisibles. Por tanto, plantea la urgencia de introducir en la enseńanza médica una concepción epidemiológica mejor y más amplia, y de preparar a los epidemiólogos con una mirada revitalizada que se abra a incorporar creativamente las amplias posibilidades que ofrece la ciencia. Semejantes reflexiones posibilitaron, en los años setenta, la aparición del concepto de "epidemiología clínica" o formación de una capacidad metodológica y rigor, tanto en la observación de casos como en la lectura de la literatura científica. Esto llevaría a lo que posteriormente se llamó "medicina basada en evidencia"(8).

\section{El rol de las comunidades}

Las comunidades tienen un papel clave en la salud, tanto preventiva como curativa, algo que vislumbró el Dr. Horwitz, haciendo notar las omisiones importantes en el origen y práctica del Servicio Nacional de Salud de Chile. Con gran claridad, afirma que se ignoró la contribución potencial de las comunidades en la identificación de los problemas, la formulación de los programas, y la ejecución y evaluación de las acciones. No bastaba, decía, con incrementar progresivamente la cobertura para garantizar el acceso efectivo de cada habitante a una unidad de salud del país; para el Dr. Horwitz, esta era una consideración insuficiente del paradigma sociobiológico de la salud, que desconocía el aporte de las ciencias sociales. 
En la sesión inaugural del Simposio Interamericano de investigaciones sobre Malaria de 1971, en El Salvador, a propósito de la falta de progresos satisfactorios en algunos de estos programas de malaria en la región, el Dr. Horwitz expresaba que: "No basta el análisis de los datos, hay que comprobar en el terreno su significado y estudiar focalmente las causas y las circunstancias que mantienen la incidencia. La enfermedad se ha hecho muy rural y para combatirla con éxito o comprenderla en su gran diversidad, profesionales y auxiliares deben estar presentes in situ (...). El problema se ha complicado por el clamor en años recientes ante el deterioro de las bellezas naturales, la destrucción de especies que queremos conservar, la contaminación indiscriminada del agua, del aire y del suelo; y los efectos que todo esto tiene sobre la salud y el bienestar de personas y comunidades. Se ha creado un verdadero síndrome de angustia colectiva con visiones del futuro que no siempre la ciencia ha podido sustanciar. Es la reacción natural de la condición humana en la época actual, que procede con frecuencia creando crisis para modificar determinadas situaciones sin precisar previamente otras consecuencias simultáneas o sucesivas que pudieran ser aún más graves"(9:513-516).

Cuatro décadas después, podemos decir que la visión del Dr. Horwitz ha sido preclara. Hoy en día no cabe duda en la región que, más allá de la cobertura de la salud, es necesario apuntar a que las mismas comunidades, con sus peculiaridades y rasgos culturales propios, identifiquen sus problemas de salud y los vacíos en la formación o en su aproximación de lo que significa la salud para cada una de ellas. De ahí que hoy es clave, primero, identificar las prácticas culturales locales, para luego poder compatibilizarlas con las prácticas globales de la salud pública derivada de la medicina basada en la evidencia.

Para Abraham Horwitz, el tema central de su periodo en la Organización Panamericana de la Salud (OPS) fue la relación entre salud y desarrollo, y lo expresaba así ya en 1960 en su artículo "Problemas de la educación para la salud en las Américas": "La salud no constituye un fin en sí misma, no vivimos únicamente para ser sanos, una de las mayores empresas de nuestro tiempo es la preservación de las culturas con el debido res- peto a las tradiciones y la forma de vida de todos los pueblos, y con la plena confianza en los valores supremos de la ética humanística" (10:313).

Entre las investigaciones a gran escala que promovió durante su mandato en la OPS, se encuentran, entre otras, la mortalidad en los adultos y luego en la niñez; este último estudio tenía como objetivo resolver importantes problemas de salud de la infancia y los primeros ańos de la niñez en América Latina y El Caribe. De hecho, entre sus logros se encuentra la caída en la mortalidad infantil a una tasa de 40 por mil en 1974.

\section{Desarrollo económico y salud}

El Dr. Horwitz estaba convencido de que no es suficiente la colaboración entre las ciencias económicas y las de la salud, si esto no se refleja en una mayor justicia social, una existencia más digna y con mayor felicidad del hombre para vivir y realizarse en plenitud. En un artículo de 1961, "Reflexiones sobre economía y salud", destacaba la vinculación existente entre el desarrollo económico y el estado de salud de la población, desde una perspectiva claramente humanista. En tal sentido dice que: "La preocupación máxima del hombre debe ser el hombre mismo, para estudiarlo y comprenderlo, con todo lo que eso implica de interés por su vida y de respeto por su esfuerzo creador. Al especialista de hoy hay que enseñar que lo importante no es saber, sino comprender, comprender al hombre, comprender el mundo, comprender su posición en la vida" (6:97).

En este mismo texto hace ver que el objeto inmediato de la medicina preventiva y curativa es el ser humano enfermo, y de modo mediato, la salud, entendida no como un fin exclusivo, sino como parte de un complejo proceso social que dé sentido y misión a la vida.

Destaca la inseparable relación entre medicina clínica, salud pública y desarrollo, ejes en los cuales siempre creyó. Es así como recalca la imperiosa necesidad de integrar la medicina individual y colectiva en el desarrollo, especialmente por el momento histórico que viven las naciones de las Américas. Afirma: "De más está decir que, dentro de nuestro propio campo de acción, debiera ya terminar el viejo conflicto entre la medicina 
preventiva y la curativa, entre Hygeia y Esculapio. Somos todos hijos de un mismo conocimiento y portadores de una experiencia que sirve un solo propósito y que debemos trasmitir perfeccionada a las próximas generaciones. Mientras más sólida la unidad, más eficiente será nuestra acción frente a las responsabilidades, porque de lo que se trata hoy es de determinar cómo podemos poner mejor nuestro esfuerzo al servicio de las familias y de los grupos sociales para luchar contra la miseria y conservar la cultura del continente" (6:102).

Incluso el Dr. Horwitz va mucho más allá, incorporando en su visión del desarrollo factores externos a la economía, lo cual en esa época significaba una perspectiva muy innovadora que se consolidaría mucho después. Advertía que el progreso social necesitaba de técnicos bien formados por las universidades, enfatizando que el desarrollo se situaba en un individuo bien preparado, no en los capitales. En el Informe Cuadrienal del Director 1962-1965, profundiza estas ideas, afirmando que en la nueva dimensión del desarrollo existen bienes no directamente medibles, probablemente no reproducibles, pero que contribuyen a la riqueza actual y potencial de los individuos y colectividades. Entre estos bienes, destaca, está la $\operatorname{salud}(11)$.

El Dr. Horwitz escribía en 1961: "El crecimiento económico depende de la concurrencia de diversos factores complementarios. Por un lado los netamente 'económicos', tales como los recursos económicos, la fuerza de trabajo, el ahorro, la inversión y la técnica; y por el otro, los 'no económicos o extraeconómicos'. Entre estos destaca la educación, la salud física y mental, la tradición científica, la capacidad investigadora, la creatividad, el espíritu de cooperación y cohesión social, el sentido de responsabilidad y disciplina colectiva"(6:99).

Coincidentemente con los esfuerzos que realizaba la OPS y el Banco Interamericano del Desarrollo (BID), para tratar en forma interrelacionada los fenómenos de salud y desarrollo, el Dr. Horwitz afirma en su artículo "Planificación del desarrollo económico y social en la América Latina" que: “(...) Es grato comprobar cómo algunos economistas, en años recientes, vienen reconociendo la calidad de la fuerza del trabajo como una forma del capital y a los servicios de salud más como inversión que como consumo" $(12: 380)^{6}$.

Este párrafo puede ser una de las primeras señales que evidencian la estrecha relación entre la OPS y el BID, donde se encontraba Felipe Herrera, también chileno. Ambos fueron precursores de la inversión en desarrollo en la región desde sus distintas posiciones ${ }^{7}$.

En forma persistente, el Dr. Horwitz coloca sus energías intelectuales en mostrar la relación indisoluble entre desarrollo y salud. Plantea, por ejemplo, respecto de la malnutrición calóricaproteica en América Latina entre niños menores de cinco años, que la carencia específica es causa frecuente de mortalidad directa por malnutrición y disminuye la resistencia a las infecciones. Dice que, "(...) la malnutrición afecta el desarrollo físico y mental de las poblaciones, condicionando rendimientos de trabajo inferiores a los normales, que a su vez repercuten en la economía de los países. A su vez la alimentación insuficiente tiene efecto sobre la conducta social de los pueblos"(12:384).

\section{Educación: importancia clave}

Los lazos entre salud y condiciones del desarrollo, han sido otro de los aspectos centrales de la obra y pensamiento del Dr. Horwitz. En su artículo "Problemas de la educación para la salud en las Américas”, publicado en 1960, expresa que la implementación de programas de intervención deben ir acompañados de una adecuada educación para asegurar el éxito, tanto a corto como mediano plazo.

Impulsa la iniciativa de evitar el aislamiento de ciertos enfermos, habilitando tratamientos externos y domiciliarios. Propone así integrar a los enfermos a la vida social.

\footnotetext{
${ }^{6}$ Jorge Jiménez de la Jara recuerda que en una conferencia de la American Public Health Association (APHA), Horwitz planteaba la paradoja a un colega estadounidense Timothy Baker que pasaba más tiempo conversando con economistas que con doctores.

${ }^{7}$ El Dr. Horwitz fue un gran impulsor de la incorporación del componente salud en los programas de formación del naciente Instituto Latinoamericano de Planificación Económica y Social (ver Teruel, J. R., 1998, mayo. Conversaciones con el Dr. Abraham Horwitz, realizada en la sede de la OPS en Washington. Transcripción, selección y edición libre, responsabilidad de los autores de este trabajo).
} 
Expresa: "Para tener éxito es preciso comprender y apoyar al paciente y sus esperanzas; también debe existir una firme resolución de llevar adelante el tratamiento aun cuando dure varios ańos. Una vez más, la educación para la salud es la llave. Se debe educar cuidadosamente a los pacientes, familias y colectividades para evitar que toda casa en que haya un enfermo sea considerada como un leprosario y se huya de ella. Para que un programa de esta clase tenga éxito, su organización necesita basarse en una minuciosa preparación de la colectividad, y esto solo puede lograrse mediante la debida educación para la salud del pueblo"(10:312).

Podemos apreciar que estas ideas que plantea el Dr. Horwitz en los años sesenta, actualmente han cobrado gran vigor. Vemos la relevancia creciente de la atención en los propios domicilios, ya sea para evitar las infecciones intrahospitalarias y darles condiciones de familiaridad a los pacientes que permitan mejorar su capacidad de recuperación, o una mejor calidad de vida en sus últimos días, respetando su voluntad, dignidad y autonomía.

También sugiere que una educación adecuada debería forjar actitudes responsables que muevan a las personas a "(...) tomar actitudes menos guiadas por sus formas culturales, tradiciones o supersticiones que impiden el acceso del público al beneficio de los nuevos métodos aportados por la ciencia" (10:309). Lo anterior no debe confundirse con la convicción del Dr. Horwitz de reconocer la dimensión cultural en la salud, rescatando al mismo tiempo los avances de la ciencia y los aportes de las tradiciones de los individuos en cada contexto cultural. Afirma que los trabajadores de la salud mundial deben conocer "(...) realmente los grupos, las colectividades y sus moldes culturales en el ambiente en que ellos se esfuerzan en aplicar sus conocimientos al servicio del público"(10:309). Enfatiza también en la red de colaboradores - hoy diríamos el trabajo en equipo- - pues, dice, mientras mayor sea esta red, mejor podrá ser la evaluación de la trayectoria de la enfermedad al interior de la comunidad.

El Dr. Horwitz establece la necesidad de hacer investigaciones en el campo de la educación de la salud, para conocer mejor las motivaciones y disímiles actitudes de las personas en distintas colectividades. Sobre la base de ese conocimiento, ob- serva, será posible combatir con mayor exactitud las propias enfermedades. Hace ver que el fracaso de ciertos programas se debe principalmente a la “(...) ineficacia en la educación, es decir, por no haberse conseguido suficiente participación, tanto de individuos como de grupos, en el empeño de ayudarse a sí mismos a alcanzar una salud mejor" (10:312). Insiste en este punto al afirmar que todos los miembros del grupo sanitario debiesen participar en el proceso educativo, durante la fase de planeamiento y a largo de la ejecución de los programas.

Vincula muy asertivamente la estrecha relación entre investigación, ciencias sociales y salud.

Dice: "La incorporación de las ciencias sociales y de la conducta y, en especial, de la psicología social, brindará a los alumnos una preparación más completa para mejor conocer las costumbres de las gentes y el medio cultural en que han de trabajar (...). Por lo tanto, resulta absolutamente esencial que la enseñanza de esta disciplina sea obligatoria en el periodo anterior a la graduación en todas las escuelas profesionales(10:313).

En dos artículos publicados en 1962, el Dr. Horwitz pone énfasis en que la salud pública es una disciplina netamente dinámica, que no puede concebirse circunscrita a un país ni a una región cuando pretende determinar los principios que la gobiernan, los métodos que emplea y los efectos que tiene en la prevención de las enfermedades, en la promoción de la salud y en la prolongación de la vida. Nos hace ver que la enseñanza en ese entonces, y tal vez también en la actualidad, pone demasiado énfasis en métodos y técnicas, al punto que el estudiante pierde la noción de las sociedades como unidades diferenciadas y diná$\operatorname{micas}(13,14)$.

En suma, su visión contiene una importante mirada sociocultural en la salud pública y promueve la incorporación de aspectos sociales en las carreras de la salud, permitiendo dar cuenta en forma mucho más adecuada de las distintas realidades en las cuales se desenvuelven las personas. Una visión que permanece vigente hasta el día de hoy. 


\section{Referencias}

1. Jiménez J. Abraham Horwitz (1910-2000) Padre de la Salud Pública Panamericana. Revista Médica de Chile 2003; 131(8).

2. Horwitz A. La nueva dimensión de la salud. Boletín de la Oficina Sanitaria Panamericana (OSP) octubre 1966; 61(4): 250-253

3. OPS. Historia de la Oficina Sanitaria Panamericana en República Dominicana. República Dominicana: OPS; 2002.

4. Larraín J. Modernidad. Razón e Identidad en América Latina. Santiago de Chile: Andrés Bello; 1996.

5. Horwitz A. La epidemiología en la América Latina. Boletín de la Oficina Sanitaria Panamericana (OSP) septiembre 1961; 51(3): 191-194.

6. Horwitz A. Reflexiones sobre economía y salud. Boletín de la Oficina Sanitaria Panamericana (OSP) agosto 1961; 51(2): 97-103.

7. Homenaje al Dr. Abraham Horwitz (1910-2000). Boletín Epidemiológico. Organización Panamericana de la Salud (BEOPS) 2000; 21(3): 2-4.

8. White K. Healing the schism: epidemiology, medicine, and the public's health. Prefacio de Halfdan Mahler, XVIII. New York: Springer-Verlag; 1991.

9. Horwitz A. Presentación del Dr. Abraham Horwitz en la sesión inaugural del Simposio Interamericano de Investigaciones sobre Malaria, San Salvador, El Salvador. Boletín de la Oficina Sanitaria Panamericana (OSP) junio 1972; 513-516.

10. Horwitz A. Problemas de la educación para la salud en las Américas. Boletín de la Oficina Sanitaria Panamericana (OSP) octubre 1960; 49(4): 309-314.

11. OPS/OMS. Informe Cuadrienal del Director, 1962-1965. La nueva dimensión de la salud. Washington, D.C.: OPS/ OMS; 1965.

12. Horwitz A. Planificación del desarrollo económico y social en la América Latina. Boletín de la Oficina Sanitaria Panamericana (OSP) noviembre 1961; 51(5): 379-386.

13. Horwitz A. Evolución de la educación médica en la América Latina. Boletín de la Oficina Sanitaria Panamericana (OSP) abril 1962; 52(4): 281-286.

14. Horwitz A. La Organización Mundial de la Salud y la Universidad en la Salud Internacional. Boletín de la Oficina Sanitaria Panamericana (OSP) mayo 1962; 52(5): 375-377.

Recibido: 3 de marzo de 2014

Aceptado: 14 de abril de 2014 